\title{
UAS Pilot Evaluations of Suggestive Guidance on Detect-and-Avoid Displays
}

\author{
Kevin Monk \\ NASA Ames Research Center \\ Zachary Roberts \\ San Jose State University
}

\begin{abstract}
Minimum display requirements for Detect-and-Avoid (DAA) systems are being developed in order to support the expansion of Unmanned Aircraft Systems (UAS) into the National Airspace System (NAS). The present study examines UAS pilots' subjective assessments of four DAA display configurations with varying forms of maneuver guidance. For each configuration, pilots rated the intuitiveness of the display and how well it supported their ability to perform the DAA task. Responses revealed a clear preference for the DAA displays that presented suggestive maneuver guidance in the form of "banding" compared to an Information Only display, which lacked any maneuver guidance. Implications on DAA display requirements, as well as the relation between the subjective evaluations and the objective performance data from previous studies are discussed.
\end{abstract}

\section{INTRODUCTION}

Unmanned Aircraft Systems (UAS), while currently limited to restricted airspace, are expected to expand across civil airspace classes within the National Airspace System (NAS) in the near future. In order to maintain safety with manned and unmanned aircraft flying alongside one another, new minimum operational performance standards are being developed by a group of subject matter experts from government, industry, and academia (RTCA, 2013; FAA, 2013). Title 14 Code of Federal Regulations section 91.113 requires pilots to "see and avoid" nearby aircraft in order to remain 'well clear' (14CFR, 2004). Since UAS pilots located at a ground control station (GCS) are unable to visually acquire potential well clear conflicts with other aircraft from an onboard cockpit, a "detect and avoid" (DAA) system that provides electronic information to the ground pilot in order to identify a threat and safely make a resolution maneuver with the GCS's command-and-control interface will be required (RTCA, 2013; Santiago \& Mueller, 2015). The minimum amount of DAA display information needed for pilots to detect potential conflicts, determine a conflict resolution, and effectively avoid losses of well clear at acceptable safety and performance levels needs to be defined.

Previous studies have sought to inform the establishment of minimum DAA display requirements. Draper, Pack, Darrah, Moulton, and Calhoun (2014) surveyed pilots on their visual information preference. The results of their survey revealed that standard intruder state information, alerting, and DAA maneuver recommendations were considered critical information elements. In simulation experiments, predictive displays that depict avoidance zones, indicators of intruder relative closest-point-of-approach (CPA), and directional icons equipped with conflict alerting have shown to be beneficial in avoiding losses of well clear and minimizing the severity of violations when they do occur (Bell, Drury, Estes, \& Reynolds, 2012; Friedman-Berg, Rein \& Racine, 2014). In addition, integrated displays with traffic information and vehicle control inputs collocated on the same monitor have been beneficial to the objective and subjective performance of pilots, especially when equipped with advanced resolution tools (Fern, Rorie, Pack, Shively, \& Draper, 2015; Santiago \& Mueller, 2015; Monk, Fern, Rorie \& Shively, 2015). Rorie \& Fern (2015) examined the distinct impact of advanced tools (e.g. auto resolutions, vector planners) from aforementioned studies and found that previously observed performance benefits were likely due in large part to the integrated, directive (i.e., a single resolution provided by a conflict resolution algorithm) nature of the maneuver guidance. The advanced tools were coupled with the GCS command-andcontrol interface and auto-populated single maneuver resolutions for pilots to upload, thus minimizing the time dedicated to determining and inputting a resolution maneuver.

The current experiment looks at the impact on pilots' subjective feedback of different presentations of suggestive maneuver guidance, which provides a range of resolution maneuvers from which a pilot can select, rather than just the single maneuver provided by directive guidance displays. In addition, all of the maneuver guidance displays were decoupled from the GCS command-and-control interface in order to represent a "minimum" implementation of the DAA system. The present study reports the subjective results of Rorie, Fern, and Shively (2016), which examined the impact of suggestive maneuver guidance on UAS pilot response times and maintenance of well clear from other aircraft.

\section{METHOD}

\section{Participants}

Sixteen active duty UAS pilots $\left(M_{\text {age }}=37\right.$ years old $)$ were recruited for the study. On average, they had 575 hours of manned flight experience in civil airspace and 1760 hours in military operations. With regard to unmanned flight experience, pilots averaged 30 hours in civil airspace and 1100 hours in military operations. 


\section{Apparatus}

Refer to Rorie et al. (2016) for an in-depth description of the experimental airspace environment, simulation architecture, and the Air Force Research Laboratory's Vigilant Spirit Control Station (VSCS; Feitshans, Rowe, Davis, Holland \& Berger, 2008) used in the present study.

$D A A$ System. The multi-level alerting structure was supplied by the Java Architecture for DAA Modeling and Extensibility (JADEM; Santiago, Abramson, Refai, Mueller, Johnson \& Snow, 2015). The color-coded alerting scheme (Table 1) was applied to all aircraft within ADS-B-like surveillance range of $15 \mathrm{~nm}$ laterally and +/- 5000 feet vertically for transponder-equipped (cooperative) intruders, and $8 \mathrm{~nm}$ laterally with the same vertical range for intruders without transponders (non-cooperative).

\begin{tabular}{|l|c|c|c|c|}
\hline \multirow{2}{*}{ Alert Level } & \multicolumn{2}{|c|}{ CPA Distance } & \multicolumn{1}{c|}{$\begin{array}{c}\text { Time } \\
\text { to Loss } \\
\text { of Well } \\
\text { Clear }\end{array}$} & \multirow{2}{*}{ Icon } \\
\cline { 2 - 4 } & Lateral & Vertical & $85 \mathrm{sec}$ & \\
\hline $\begin{array}{l}\text { DAA } \\
\text { Proximate }\end{array}$ & $<1.5 \mathrm{NM}$ & $<1200 \mathrm{FT}$ & $85 \mathrm{sec}$ & \\
\hline $\begin{array}{l}\text { Preventive } \\
\text { DAA }\end{array}$ & $<1.0 \mathrm{NM}$ & $<700 \mathrm{FT}$ & $75 \mathrm{sec}$ & \\
\hline $\begin{array}{l}\text { Corrective } \\
\text { DAA }\end{array}$ & $<0.75 \mathrm{NM}$ & $<400 \mathrm{FT}$ & 7 \\
\hline $\begin{array}{l}\text { DAA } \\
\text { Warning }\end{array}$ & $<0.75 \mathrm{NM}$ & $<400 \mathrm{FT}$ & $25 \mathrm{sec}$ & \\
\hline
\end{tabular}

Table 1. The multi-level alert thresholds and symbology.

\section{Experimental Design}

The present study examined pilots' subjective feedback on four separate DAA display configurations: Information Only (Info Only), No-Fly Bands, Omni Bands, and Vector Planner. Each display provided varying levels of suggestive DAA guidance information on the TSD for conflict resolution maneuvers.

Info Only. The Info Only display condition contained the minimum set of intruder state information, which included its call sign, range, location, heading, bearing, relative and absolute altitude, vertical trend, and ground speed. This information, along with the conflict alerting logic, was available in all conditions. There was no maneuver guidance provided in this configuration.

No-Fly Bands. The No-Fly Bands condition, generated by the Stratway+ DAA system (Hagan, Butler \& Maddalon, 2011), displayed yellow bands around headings and vertical speeds that would cause an eventual Preventive DAA, Corrective DAA, or DAA Warning alert (Figure 1). The conflict prediction was based on the current state of ownship, and did not take into account future changes in the flight plan. There were no bands presented at regions that were considered safe or not probed. Pilots were trained to maneuver outside of the No-Fly banding in order to maintain well clear from nearby aircraft. When well clear was lost and pilots could no longer remain outside of the well clear threshold, dashedgreen recovery bands were presented as an additional aid in maximizing their separation as quickly as possible.

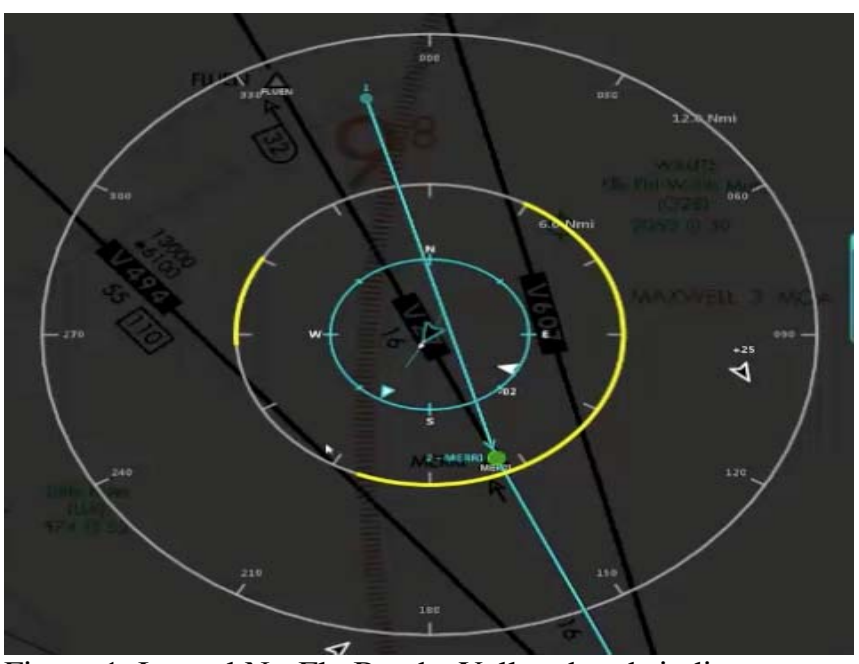

Figure 1. Lateral No-Fly Bands. Yellow bands indicate headings that would result in a DAA conflict alert if flown.

Omni Bands. The Omni Bands, generated by the JADEM DAA system, presented bands that probed the safety of specific heading and altitude options (Figure 2). Altitudes within 1,500 ft. of ownship were presented in place of the vertical speed indicator at $500 \mathrm{ft}$. increments. The bands used in this configuration took into account ownship intent, and applied banding colors that corresponded with the predicted alert type. Safe altitude and headings that would not result in the onset of a DAA conflict alert were indicated with green banding. Dashed yellow bands indicated an eventual Preventive DAA alert in the region, solid yellow bands indicated an eventual Corrective DAA alert, and red bands were presented in regions that would lead to a DAA Warning absent any pilot action. Pilots had to avoid the solid yellow and red banding in order to maintain well clear.

Vector Planner. In the Vector Planner configuration, which was also generated by the JADEM DAA system, pilots were required to manually probe heading and altitude options with the vector planning tools (Figure 3). Altitude options were displayed in $500 \mathrm{ft}$. increments, and clicking an option in the altitude table would trigger the vertical maneuver guidance in the Omni Bands condition for that specific altitude only. To test specific headings, pilots had to click and drag a vector arrow, which changed colors according to the predicted alert level at each degree. A red arrow indicated an eventual DAA Warning, a solid yellow arrow indicated an eventual Corrective DAA alert, and a hollow yellow arrow indicated an eventual Preventive DAA alert. A green arrow or altitude border indicated the option would maintain separation. The vector arrow and altitude options were always grey, similar to the Info Only condition, unless manually activated by the pilots. 


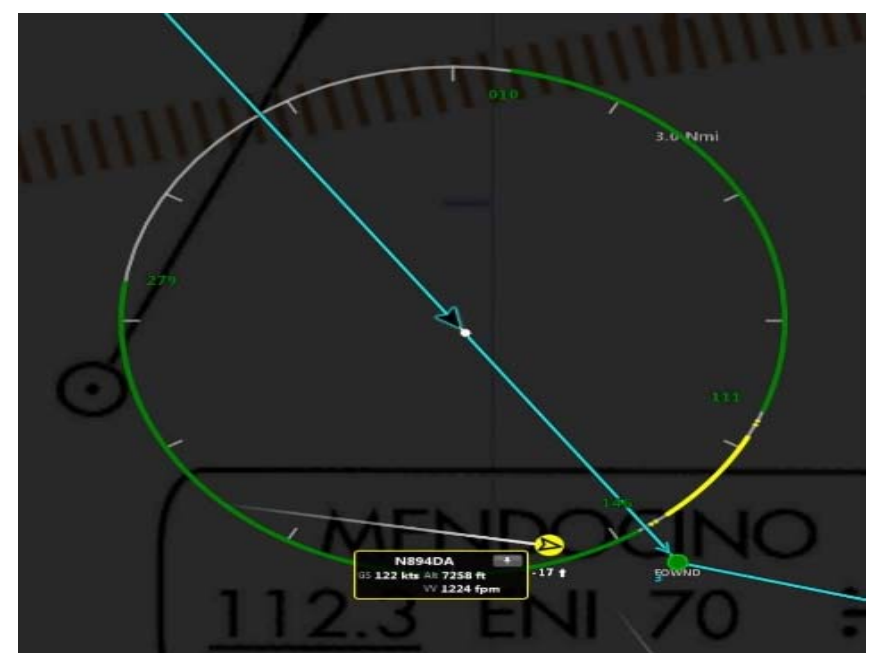

Figure 2. Lateral Omni Bands during an active Corrective DAA alert. Green bands indicate safe headings that would resolve the alert if flown.

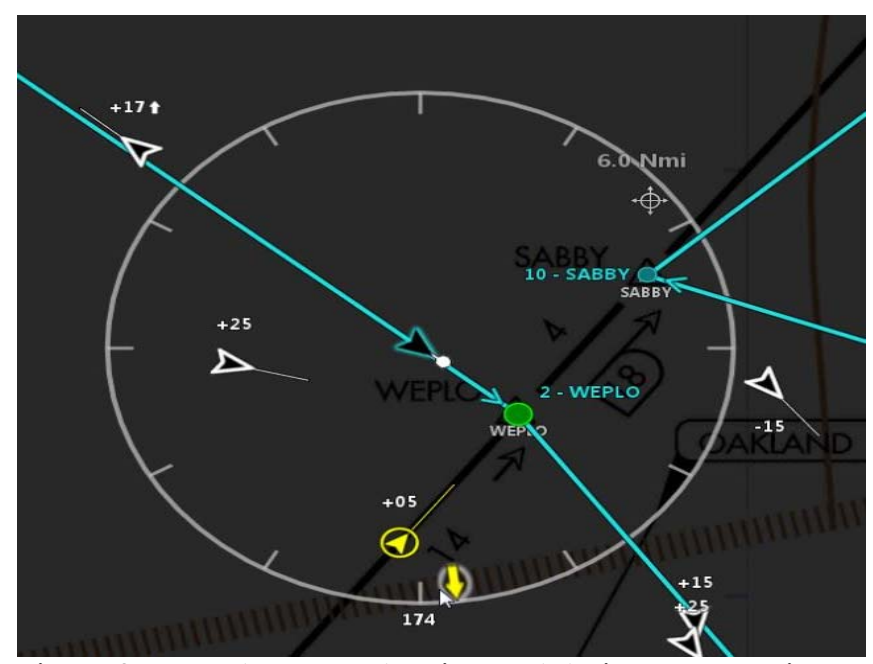

Figure 3. Lateral Vector Planning tool during a Preventive DAA alert. Solid yellow arrow indicates the specific heading selected $\left(174^{\circ}\right)$ will lead to a Corrective DAA alert if flown.

\section{Procedure}

Training. Once demographics and informed consent forms were completed, pilots received training on VSCS functionality and vehicle control inputs. The basic function training continued until pilots showed proficiency in maneuvering the aircraft and performing secondary tasks. Pilots were then trained on the DAA alerting structure shown in Table 2, and were given test encounters with traffic displayed on the TSD. Training on each display configuration was given before it was presented in an experimental trial. It consisted of a slide presentation and hands-on demonstration, followed by a 20 -minute practice session.

DAA Pilot Task. Pilots completed a 37-minute scenario with each of the four display configurations. The order of presentation was counterbalanced between participants. The primary task was to navigate a simulated MQ-9 Reaper along a pre-filed flight path while maintaining well clear from nearby traffic. There were nine scripted encounters in each scenario, six of which predicted to cause a loss of well clear with ownship if the pilot failed to maneuver around the intruder. All maneuver guidance available on the TSD was independent of the GCS command-and-control interface, so pilots were required to manually input all maneuvers when resolving conflicts. Pilots were trained to coordinate their maneuvers with a confederate air traffic controller, time permitting. A series of secondary tasks (e.g. responding to mission-related chat messages and completing electronic checklists) were also included as part of the experiment.

\section{MEASURES}

\section{Post-Trial Questionnaire}

Following each experimental trial, participants completed a post-trial questionnaire with subjective ratings pertaining to the preceding display configuration.

Task Performance. Pilots rated their "ability to handle all pilot tasks" on a 5-point scale (1 - Unacceptable to 5 Excellent).

Ease of Use. For each display configuration, pilots rated their agreement to the statement "This display was easy to use" on a 5-point Likert scale (1 - Strongly Disagree to 5 Strongly Agree).

Conflict Assessment and Avoidance. Pilots rated their agreement with statements about whether each display provided the necessary information to "predict a potential loss of well clear" and "perform a maneuver for well clear maintenance" on a 5-point Likert scale (1 - Strongly Disagree to 5 - Strongly Agree).

Trust. Pilots rated their agreement to the statement, "I trusted the accuracy of the information provided by the display" on a 5-point Likert scale (1 - Strongly Disagree to 5 - Strongly Agree).

\section{Post-Simulation Questionnaire}

Following the final experimental trial, a post-simulation questionnaire queried the pilots' agreement with a number of statements regarding the alerting logic, as well as preferences for airspace display information location and advanced display features.

Display Configuration Preference. Pilots responded to the statement, "Rank the following display configurations in order of their effects on your ability to maintain well clear". Each display configuration received one of four rankings (1 - Best Supported to 4 - Least Supported). Pilots were also asked to provide ratings about how each suggestive display affected their "ability to maintain well clear, compared to Info Only" on a 5-point scale (1 - Significantly Worse to 5 - Significantly Better).

\section{RESULTS}

Pilot responses to post-trial and post-simulation questionnaires were analyzed across the four display configurations using a one-way repeated measures Analysis of Variance (ANOVA). Greenhouse-Geisser corrections are 
reported in analyses where Mauchly's sphericity test was significant. An alpha level of .05 was used across all analyses.

\section{Post-Trial Questionnaire}

Task Performance. Display configuration had a significant effect on pilots' subjective assessment of their ability to handle DAA tasks, $F(3,45)=7.11, p=.001$. Pilots indicated higher ability to handle all pilot tasks with the Omni Bands $(M=4.44, S E=0.14)$ and No-Fly Bands $(M=4.44, S E$ $=0.13)$ displays compared to the Info Only $(M=3.94, S E=$ $0.14)$ and Vector Planner displays $(M=3.69, S E=0.18)$. No differences in perceived task performance between the banding displays were found, $p>.05$.

Ease of Use. There was a significant main effect of display configuration on ease of use ratings, $F(1.73,25.99)=$ $9.70, p=.001$. Pilots indicated the Omni Bands display $(M=$ 4.75, $S E=0.11)$ was easier to use than the Info Only $(M=$ $3.88, S E=0.24)$, No-Fly Bands $(M=4.13, S E=0.24)$, and Vector Planner $(M=2.88, S E=0.36)$ displays. Additionally, the Info Only display $(M=3.88, S E=0.24)$ was rated easier to use than the Vector Planner display $(M=2.88, S E=0.36)$.

Conflict Assessment and Avoidance. There was a significant main effect of display configuration on perceived conflict assessment, $F(3,45)=12.35, p<.001$ (Figure 4). Higher agreement ratings with regard to prediction of potential losses of separation were given for the Omni Bands display $(M=4.69, S E=0.12)$ compared to the Info Only $(M=3.13$, $S E=0.27)$ and Vector Planner $(M=3.19, S E=0.29)$ displays. The No-Fly Bands display $(M=4.44, S E=0.24)$ also received higher agreement ratings than the Info Only display $(M=3.13$, $S E=0.27$ ). No differences in agreement ratings for conflict assessment were found between the No-Fly Bands and Omni Bands displays, $p>.05$.

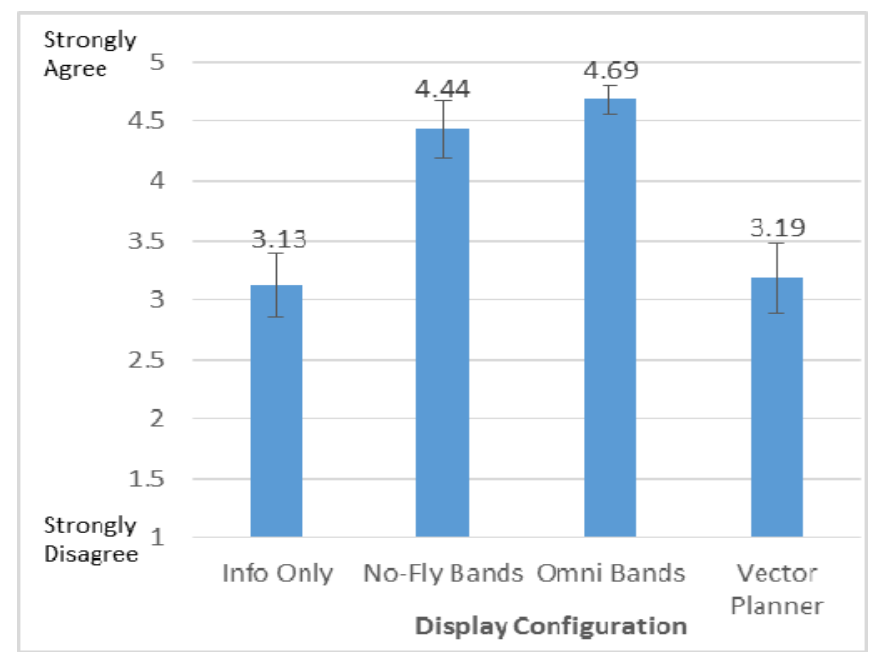

Figure 4. Adequacy of information for conflict assessment by display configuration.

There was also a significant main effect of display configuration on perceived conflict avoidance, $F(3,45)=$ $14.46, \mathrm{p}<.001$. Higher agreement ratings with regard to avoidance of potential losses of separation were given for the Omni Bands $(M=4.69, S E=0.12)$ and No-Fly Bands $(M=$
4.50, $S E=0.20)$ displays compared to the Info Only $(M=$ 2.94, $S E=0.25)$ and Vector Planner $(M=3.25, S E=0.30)$ displays. No differences in agreement ratings for their ability to perform separation maneuvers were found between the Omni Bands and No-Fly Bands displays, $p>.05$.

Trust. There was no significant difference in the ratings of trust in the accuracy of information between display configurations, $p>.05$. The Vector Planner display $(M=3.88$, $S E=0.29)$ was the only display configuration that did not average at least slight agreement with the statement.

\section{Post-Simulation Questionnaire}

Display Configuration Preference. Pilots ranked the Omni Bands display as the most beneficial for maintaining well clear; it was ranked first by 14 of 16 pilots $(88 \% ; M=$ 1.19). The No-Fly Bands display received the second most favorable rankings, with 10 of 16 pilots $(63 \%)$ indicating it was the second best display $(M=2.19)$. While the Vector Planner display was ranked second by 4 pilots $(25 \%)$, it also received the most last place votes $(50 \%)$ and had the lowest average ranking overall $(M=3.25)$. While the Info Only display did not receive the most last place rankings, only one pilot ranked it as a top-two display $(M=3.38)$. Furthermore, the Omni Bands $(M=4.81)$ and No-Fly Bands $(M=3.81)$ displays were both rated significantly and somewhat better at aiding ability to maintain well clear than the Info Only display, respectively, while the Vector Planner display $(M=$ 2.56) was the only to be rated worse than the Info Only display (Figure 5).

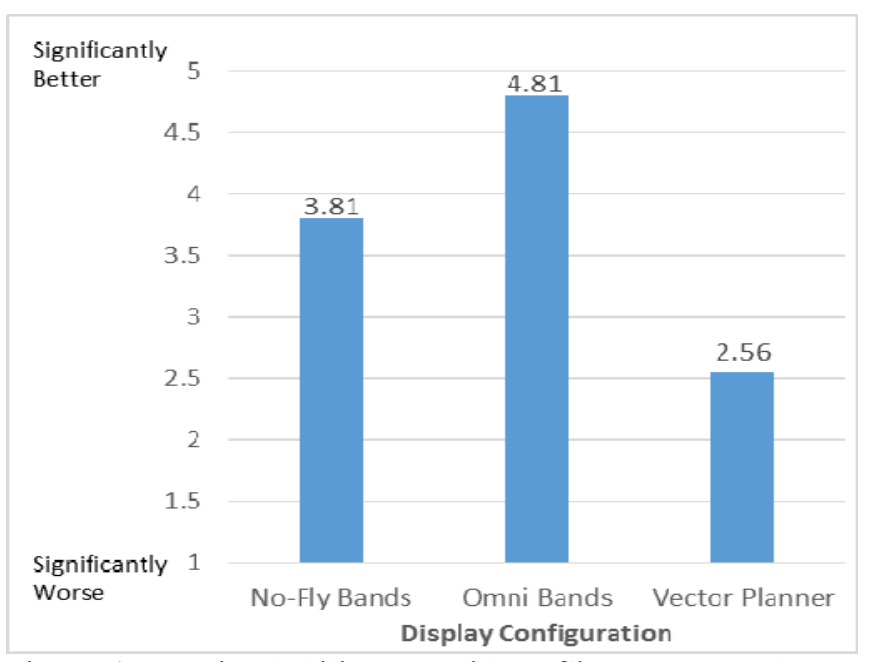

Figure 5. Perceived ability to avoid conflicts (compared to Info Only) by display configuration.

\section{DISCUSSION}

The above results suggest that suggestive maneuver guidance is highly favored by pilots - even when it is not integrated with command-and-control interface, and especially when it is visible on the display at all times. Both banding displays that had suggestive maneuver guidance for altitudes and headings available by default received the highest subjective ratings on overall task performance and ability to 
detect and avoid conflicts compared to the Info Only and Vector Planner displays. The banding displays were also nearly unanimously voted as the most preferred. This is consistent with the objective findings observed by Rorie et al. (2016), which revealed significantly quicker response times with the banding displays compared to Vector Planner and Info Only. The Omni Bands display was most preferred, and voted easiest to use. The indication of threat severity and future flight states being taken into account may have added to the predictive nature of the guidance.

The Vector Planner display did not yield much more favorable results compared to Info Only, even though it contained an element of suggestive guidance. While the Vector Planner was capable of providing the same type of guidance as the more favored Omni Bands (i.e., the predicted safety level of potential headings and altitudes), the fact that pilots had to manually engage the function likely detracted from its usefulness. It was essentially an Info Only display until the pilot activated the guidance, which only remained visible for five seconds after engagement. Rorie et al. (2016) found significantly slower edit times associated with the Vector Planner display, which suggests that pilots spent a long time probing individual headings and altitudes when they opted to do so. Although the Vector Planner tool was optional and provided a specific resolution value as opposed to the broad resolution range in the banding displays, it was the only display that was not rated easy to use. It should be considered that the suggestive guidance provided by the banding displays were more dynamic, as they were constantly visible, updating to reflect any changes to ownship or intruder states. The added action of engaging the Vector Planner tool may have complicated the DAA task to an extent that only added to pilots' workload.

While suggestive maneuver guidance in the form of banding may not be an absolute minimum requirement, it has demonstrated true benefits, both objective and subjective, for pilots performing DAA tasks. It must be considered that not all guidance displays are equal; a display with guidance shown in a manner similar to Vector Planner may fare worse than no guidance at all if not implemented well. Subsequent research is needed to further define minimum information requirements for DAA displays, with considerations made to interoperability with existing collision avoidance systems and variations in aircraft performance, airspace environment (e.g. weather), and navigation interfaces.

\section{REFERENCES}

Bell, S., Drury, J., Estes, S., \& Reynolds, C. (2012). GDTI: A ground station display of traffic information for use in sense and avoid operations. Digital Avionics Systems Conference (DASC), 2012 IEEE/AIAA $31^{\text {st }}$, Williamsburg, VA.

Code of Federal Regulations, 14 CFR, Part 91, Sec. 91.113 (2004).

Draper, D.H., Pack, J.S., Darrah, S.J., \& Moulton, S.N. (2014). Human-machine interface development for common airborne sense and avoid program. Proceedings of the Human Factors and Ergonomics Society $58^{\text {th }}$ Annual Meeting, Chicago, IL.
Federal Aviation Administration (FAA). (2013). Integration of civil UAS in the NAS roadmap, first edition. FAA, Washington, D.C.

Feitshans, G. L., Rowe, A. J., Davis, J. E., Holland, M., \& Berger, L. (2008). Vigilant spirit control station (VSCS) - 'The face of COUNTER'. Proceedings of AIAA Guidance, Navigation and Control Conf. Exhibition, Honolulu, HI.

Fern, L., Rorie, R.C., Pack, J., Shively, R.J., \& Draper, M. (2015). An evaluation of detect and avoid (DAA) displays for unmanned aircraft systems: the effect on information level and display location on pilot performance. Proceedings of the $15^{\text {th }}$ Annual AIAA Aviation, Technology, Integration and Operations Conference, Dallas, TX.

Friedman-Berg, F., Rein, J. \& Racine, R. (2014). Minimum visual information requirements for detect and avoid in unmanned aircraft systems. Proceedings of the Human Factors and Ergonomics Society $58^{\text {th }}$ Annual Meeting, Chicago, IL.

Hagen, G., Butler, R., \& Maddalon, J. (2011). Stratway: A modular approach to strategic conflict resolution. Proceedings of 11th AIAA Aviation Technology, Integration, and Operations (ATIO) Conference, Virginia Beach, VA.

Monk, K., Fern, L., Rorie, R.C., \& Shively, R.J. (2015). Effects of display location and information level on UAS pilot assessments of a detect and avoid system. Proceedings of the Human Factors and Ergonomics Society 59th Annual Meeting, Los Angeles, CA.

Rorie, R.C. \& Fern, L. (2015). The impact of integrated maneuver guidance information on UAS pilots performing the detect and avoid task. Proceedings of the $59^{\text {th }}$ Human Factors and Ergonomics Society Annual Meeting, Los Angeles, CA.

Rorie, R. C., Fern, L., \& Shively, J. (2016). The impact of suggestive maneuver guidance on UAS pilot performing the detect and avoid function. AIAA Infotech@ Aerospace, San Diego, CA.

RTCA. (2013). Terms of reference RTCA special committee 228 minimum performance standards for unmanned aircraft systems. RTCA Inc., Washington, DC.

RTCA, Inc. Special Committee 228 (2013). Detect and avoid (DAA) whitepaper. RTCA-SC228-WG1-WP01-01.

Santiago, C., Abramson, M., Refai, M., Mueller, E., Johnson, M., \& Snow, J. (2015). Java architecture for detect and avoid (DAA) modeling and extensibility (JADEM). NASA/TM-2015-TBD, unpublished.

Santiago, C. \& Mueller, E.R. (2015). Pilot evaluation of a UAS detect-and-avoid system's effectiveness in remaining well clear. 11th USA/Europe Air Traffic Management Research and Development Seminar, Lisbon, Portugal. 